\title{
Analytical study of the lane-by-lane variation for lane-utility and traffic safety performance assessment using NGSIM data
}

\author{
Zhandong Xu \\ School of Transportation and Logistics, Southwest Jiaotong University, 610031 Chengdu, China
}

\begin{abstract}
Highlights the shortcoming of using aggregate data as a whole with the assumption of similar lane-usepattern over lanes, traffic variations are attentively investigated to explore traffic dynamics distribution both in laneutility in efficiency and safety performance lane-by-lane. In this paper, above two dimensions are examined for each of five mainline lanes on Highway 101 in Angeles using Next Generation Simulation (NGSIM) data. The lanespecific data is statistically processed to reflect lane utility in lane flow parameters along the location and identify Lane Risk Coefficient by verifying degree of consistence vehicles operation with 'Assured Clear Distance Ahead' criterion. It appears completely new that the most-middle lanes are similar utilized under congested conditions compared to two sides of the lanes, as well as the quite close values of LRC to reveals an uniformly safety level between Lane 2 and Lane 3. The findings of this study are intended to be relevant with the calibration of microscopic simulation models and suggest implementing a proper variable speed toward under-utilized shoulder Lane 5 but with worst safety performance.
\end{abstract}

\section{Introduction}

It is commonly recognized that macroscopic traffic dynamics acquiesce in strong assumption that all lanes behave with uniformity of traffic flow variables, such as flow, density and speed that represent the average across all available lanes. However, critics have also argued that a complete traffic flow theory must take into account lane specific behaviour because it affects capacity and transitions between uncongested and congested traffic flows. Highlights the shortcoming of using aggregate and average data, non-equilibrium over lanes can occurs and fails to identify or forecast different traffic states without a distinction over lanes [1]. In addition, the Highway Capacity Manual (HCM, 1994) acknowledges the inherent error for neglecting the variation of each lanes and aggregating them in the 2010 edition, which explicitly indicating that the traffic distribution of a multi-lane roadway depends on a number of factors such as traffic regulation, traffic composition, driving behaviour and the origin-destination patterns [2].

Other studies have been extensively focus on the lane flow distribution (LFD) to statistically explore the relative impacts of lane-by-lane patterns with location and daily variability and examine the lane variability across lanes [3]. Their studies against the lane-specific variability typically confirmed the relationship between traffic flow parameters across lanes using curve fitting techniques. However, most researches focus on an equilibrium traffic flow without the influence of merg ing traffic and safety consideration. This raises the problem how lane-utility changes along the location of roadway with merging flow.

Based on which, quite a few of researches investigated whether a vehicle is in consistent with Assured Clear Distance Ahead (ACDA) criterion for safety performance assessment [4]. It is confirmed that unbalanced lane-using patterns will result in variability of safety performance of vehicles operating on different lanes. However, little research has been done to investigate safety performance among several available lanes at a stretch of freeways. Thus, this paper intends to assess the safety coefficient across different lanes considering the lane variation on freeway. In order to do so, there are two aspects of lane use pattern have to be analysed. The first one is to statistically verify significant variation across lanes using detected data from the Next Generation Simulation (NGS IM) program. The second one is to assess the vehicle operation and safety performance on each individual lane and makes an evaluation lane-by-lane.

This paper is organized as follows. Section 2 presents the studied freeway area and a detail description of data items. Section 3 present the lane traffic distribution along the driveway, this is followed by analys is of lane-by-lane safety performance assessment in Section 4. This paper concludes by Section 5 with the implication of the results.

\section{Site and data}

This study focused on the program of the Next Generation Simulation (NGSIM) southbound direction of 
U.S. freeway 101 (Hollywood Freeway) as illustrated in Figure 1. The freeway 101 is located in Angeles, California and mounted with video cameras for record ing vehicle trajectory dataset. The site is approximately $640 \mathrm{~m}$ in length with five mainline lanes, one-way throughout the section including the on-ramp at Ventura Boulevard and the off-ramp at Cahuenga Boulevard. We named the five main line lanes from leftmost to the right as Lane 1 to Lane 5.

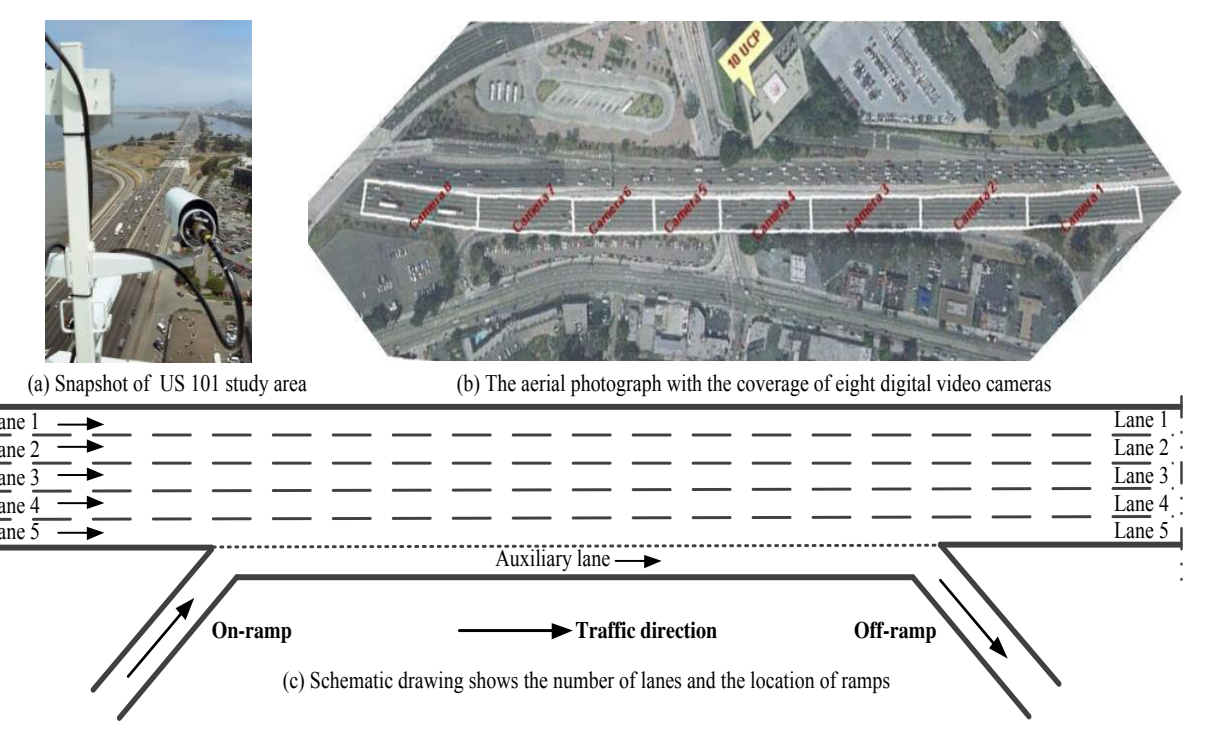

Figure 1. Traffic data collection via cameras on a section of about $640 \mathrm{~m}$ on US Highway 101 .

The program has collected high-quality and lanespecific thousands of vehicle paths in a total of 45 minutes between 7:50 a.m. and 8:35 a.m. on June 15, 2005. Further, the selection of this time frame determines the study focused on experienced heavy congestion (LOS E). Video measurements of individual vehicle dynamics including vehicle position (coordination $x-y$ ), vehicle length, occupied lane and etc., were recorded at every 0.1 second, ensuring the purpose of this study using lanespecific data.

\section{Lane traffic analysis}

Traditionally, the capacity and service quality of freeway is evaluated by using the entire freeway traffic data, neglecting the capacity distribution of more precisely traffic variations between different lanes. Not many speculations from lane-level view are raised to analyse the lane-using patterns and distributions across lanes. In this paper, the three microscopic traffic flow parameters, including traffic flow, speed and occupancy, are investigated to explore lane-level capacity distribution.

\subsection{Traffic flows}

The single direction of studied freeway consists of five mainline lanes, and a total of 7496 vehicles in 45 minutes are statistically added up from the classification of three types of traffic composition (i.e. small car and truck) and traffic demand. According to the whereabouts of the traffic flow without considering zero ramp-to-ramp traffic, it can be divided into three categories: freeway-tofreeway traffic, freeway-to-ramp traffic and ramp-tofreeway traffic. The traffic flow rate per lane can be expressed as the number of vehicles per hour occupied that lane through the section. Table 1 summarizes statistical calculation results of traffic flow across lanes.

Table 1. Vehicle types and traffic demand distribution among individual lanes.

\begin{tabular}{cccccc}
\hline & \multicolumn{2}{c}{ Type (veh) } & \multicolumn{3}{c}{ Traffic Demand (veh) } \\
& Car & Truck & $\begin{array}{c}\text { Freeway } \\
\text { to } \\
\text { freeway }\end{array}$ & $\begin{array}{c}\text { Freeway } \\
\text { to } \\
\text { ramp }\end{array}$ & $\begin{array}{c}\text { Ramp } \\
\text { to } \\
\text { freeway }\end{array}$ \\
\hline Lane1 & 1219 & 7 & 1218 & 1 & 7 \\
Lane2 & 1466 & 4 & 1437 & 2 & 31 \\
Lane3 & 1492 & 13 & 1426 & 2 & 77 \\
Lane4 & 1541 & 47 & 1388 & 11 & 189 \\
Lane5 & 1638 & 69 & 1085 & 223 & 399 \\
\hline Total & 7356 & 140 & 6554 & 239 & 703 \\
\hline
\end{tabular}

- Outer lane carries more flows This is because of origin-to-destination purposes, which is inevitable for those vehicles to use. Meanwhile, heavier vehicles (e. g. truck or bus) using the rightmost lanes not only for route patterns, also corresponding to their less-powerful to be overtaken and mass-loaded characters so as to suggest their driving preference at outer lane.

- Freeway-to-freeway vehicles tend to use the leftmost lanes to avoid those turning vehicles, in contrast to most of merging vehicles driving on Lane 4 and Lane.

In general, it can be clearly observed that the traffic flows on median Lane 1 and shoulder Lane 5 are significant different with adjacent lanes, with $19.88 \%$ and $7.5 \%$ of growth respectively. The traffic flow per lane of lane-using variation reflects the traffic flow parameters do not have uniform volumes across available lanes. Specifically speaking, different characteristics of roadway, such as traffic composition, traffic regulation 
and rules, driving behavior and so forth will be the causative factors for such traffic flow distribution.

\subsection{Traffic speed}

As Kerner (2004) argues that not all roadway capacity is used as long as the traffic speeds are not equal across lanes, which implies non-equilibrium of speed over lanes is harmful to total flows [6]. The sensors have collected instantaneous velocity of vehicles at every $0.1 \mathrm{~s}$ in traffic dataset. Time mean speed (TMS) for vehicle $k$ is calculated considering $n$ midpoints where the vehicle travelling on it, we will have the following:

$$
\overline{u_{k}}=\frac{1}{n} \sum_{t}^{n} v\left(t_{k}\right)
$$

The TMS corresponding to each lane is segmented to obtain basic statistic such as mean, standard deviation, median and skewness, as summarized in Table 2.

Table 2. Basic statistics of TMS for different lanes.

\begin{tabular}{lcccc}
\hline & $\begin{array}{c}\text { Mean } \\
(\mathbf{k m} / \mathbf{h})\end{array}$ & $\begin{array}{c}\text { Standard } \\
\text { Deviation } \\
(\mathbf{k m} / \mathbf{h})\end{array}$ & $\begin{array}{c}\text { Median } \\
(\mathbf{k m} / \mathbf{h})\end{array}$ & $\begin{array}{c}\text { Skew- } \\
\text { ness }\end{array}$ \\
\hline Lane 1 & 33.49 & 10.93 & 31.76 & 1.42 \\
Lane 2 & 36.67 & 11.75 & 35.22 & 0.74 \\
Lane 3 & 37.01 & 12.13 & 35.45 & 0.69 \\
Lane 4 & 37.74 & 12.03 & 36.29 & 0.54 \\
Lane 5 & 39.56 & 10.92 & 39.30 & 0.14 \\
\hline
\end{tabular}

From the table it can be seen that the mean and median value of TMS show an increasing trend from leftmost lane to another. And the standard deviation is roughly $11.5 \mathrm{~km} / \mathrm{h}$. The decrease in positive values of skewness with outer lane position indicates that the distribution of TMS is more skewed toward the right at the leftmost Lane 1. To statistically confirm the difference of TMS between two adjacent lanes, hypothesis testing using Kolmogorov-Smirnov Test is performed to prove speed adjustment variation of drivers across lane. The null and alternate hypothes is is defined as follows:

$H_{0}$ : The distribution of TMS is no different between two adjacent lanes.

$H_{l}$ : The distribution of TMS is different between two adjacent lanes.

The significant level of hypothesis test is set to be 0.95. The results including the KS-Statistic and P-Value are given in Table 3 .

Table 3. Hypothesis test for TMS between adjacent two lanes.

\begin{tabular}{lccc}
\hline & KS-Statistic & P-Value & Hypothesis \\
\hline Lane 1 \& Lane 2 & 0.182 & $\rightarrow 0^{+}$ & Reject $\mathrm{H}_{0}$ \\
Lane 2 \& Lane 3 & 0.038 & 0.230 & Accept $\mathrm{H}_{0}$ \\
Lane 3 \& Lane 4 & 0.072 & 0.011 & Reject $\mathrm{H}_{0}$ \\
Lane 4 \& Lane 5 & 0.140 & $\rightarrow 0^{+}$ & Reject $\mathrm{H}_{0}$ \\
\hline
\end{tabular}

Note: $\rightarrow 0^{+}$: Infinitelyclose to 0 in positive.

The results of the hypothesis test indicate that the distribution of Lane 2 and Lane 3 is indeed small variations than other adjacent lanes. We conduct a range of KS-Tests for each lane to examine likelihood statistic hypothes is, and the results demonstrate that TMS accords best fitting with lognormal distributions of Lane 1-4 and normal distribution of Lane 5, which is shown in Figure 2.

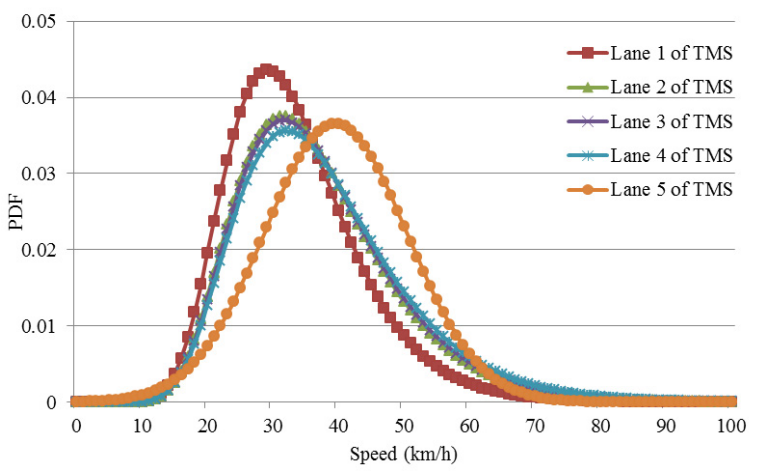

Figure 3. Best fitted distributions for TMS of five lanes.

From Figure 3, it can be seen that as lane location closing to the right the highest expected outcome decreased and gradually stabilized to $3.6 \%$. It can also be observed that the distribution are increasingly skewed to the right with lane position coming towards the left, which confirms that an increasing proportion of drivers have a tendency to slow down to be more prudent. The figure also demonstrates distributions of three middlemost lanes have tiny variation reciprocally, and relatively great variety arises at two bilateral lanes. However, the higher averaged speed on Lane 5 indicate drivers to behave more aggressive with merging traffics, which is harmful to cause hidden dangers.

\subsection{Occupancy}

Time occupancy $\rho$ is defined as the percent of time vehicles occupy the section to the total detected time interval, which can directly reflects the lane-utility along the location. To formally describe the considered traffic density of section along each lane, we denote $\rho_{i, x}$ to represent time occupancy at the location of $x$ in $i^{\text {th }}$ lane. If the vehicle $k$ has passed through $x$ of $i$ and $l_{k}$ is the length of subjective vehicle $k$, the elapsed time will be added up to calculate final observing $\rho_{i, x}$ with equation (2), and illustrated in Figure 4.

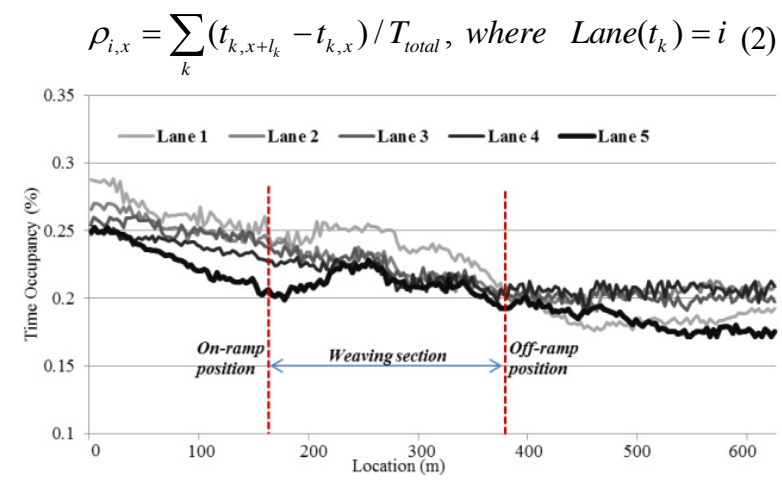

Figure 4. Time occupancy along the carriageway over lanes.

As we can clearly observe from Figure 4, the values of time occupancy range between $15 \%$ and $30 \%$ for five 
lanes considered. The fluctuation within a narrow range is corresponding with vehicles lane-changing behavior and possibly inaccurate positioning errors.

The figure also confirms that an unbalanced laneutility exists across lanes. As for median Lane 1: the occupancy has been at a relatively high level at upper reaches with slower speed, on which may result in semicongestion. Thereby, more motorists will aspire to higher speed travelling at lower density lane, which can account for the sharp decline trend. The middle of three lanes (i. e. Lane 2, Lane 3 and Lane 4) has little variations reciprocally: the curves show a closing to linear decreasing trend (a total of $6 \%-7 \%$ ) with approaching downstream to off-ramp position, and after through that the time occupancy keeps maintain ing at nearly $20 \%$. The occupancy on shoulder Lane 5: decreases (nearly 5\%) from orig in to on-ramp position, which is because drivers tend to change to the left lanes making room near the onramp. The succedent increasing with $3 \%$ indicates a considerable proportion of vehic les merg ing onto the lane.

\subsection{Findings}

The statistical results demonstrate that traffic condition at the same period of time vary across available lanes. In conference to these past studies at heavy traffic flow, we have similar conclusions to some extent that the shoulder Lane 5 is under-utilized compared to inner lanes with higher occupancy. And the median Lane 1 carries a large part of occupancy at congestion flow. Once a certain degree of congestion has set in, the prohibition of overtaking at left-side no longer stands and the traffic in three middle lanes become almost equal regardless of whether in flow, speed or density.

By analysing traffic flow lane-by-lane, we have found that the median Lane 1 is utilized most and shoulder Lane 5 is just the opposite in congested condition. The processes demonstrate no difference between Lane 2 and Lane 3 and imply equilibrium of lane use of the traffic using Kolmogorov-Smirnov analysis. In addition, comparing to inner lanes without being influenced by merging flow, the extremely unstable but aggressive vehicles at Lane 5 are likely to introduce hidden danger along the driveway. The assessment of such lane-by-lane safety performance is conducted in next section.

\section{Safety performance assessment}

As is stated above, traffic flow distributions are destined to make a difference on driving behaviors in real traffic environment, further causing the non-equilibrium of safety performance across lanes. However, few previous studied have been able to draw on structured research into the opinions and attitudes of safety performance evaluation at lane-level view, not to mention exploring impacts of lane flow on safety performance variability. In this section, we will extend speed-based rule to consider safety performance of motorists for individual lane, generally known as the Assured Clear Distance Ahead Rule (ACDA).

\subsection{Methodology}

The ACDA rule requests drivers operate their vehicle at a limited speed and sufficient spacing from the vehicle ahead by the objective purpose to ensure collision avoidance. In other words, the safety operation of an ego vehicle is capable to be identified whether drivers follow the ACDA criterion along the driveway, beware of future hazard behavior of the preceding vehicle may suddenly slow down or even stop.

Considering two vehicles, the following vehicle $v e h_{f}$ and the preceding vehicle $v e h_{p}$, are travelling on the same lane. Without loss of generality, we are focusing on extreme safe operation of vehic les in the driveway so that we simply assume the maximum permitted deceleration $a_{\text {max }}$ for all vehicles. Assuming that veh $h_{p}$ unexpectedly brakes at the constant maximum negative acceleration from the beginning of braking process and the following vehicle continues driving with constant velocity $v_{f}$ before fully brakes after a reaction delay time $\varepsilon$. We denote $\Delta x_{0}$ as the initial spacing gap of two vehicles and $l_{p}$ as the length of preceding vehicle. The stopping distance $x_{p}$ and $x_{f}$ that vehicles travel during braking from initial velocity to zero are obtained by general kinematic solution,

$$
\left\{\begin{array}{l}
x_{p}=\frac{1}{2} \frac{v_{p}^{2}}{a_{\max }{ }^{2}} \\
x_{f}=v_{f} * \varepsilon+\frac{1}{2} \frac{v_{f}^{2}}{a_{\max }{ }^{2}}
\end{array}\right.
$$

We introduce the risk factor $\varphi$ to investigate whether

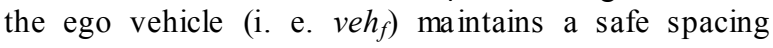
distance and how hazard condition it would be when the front vehicle stops suddenly, which can be written as,

$$
\varphi\left(t, v_{e h}, v_{e} h_{p}\right)=\frac{x_{f}+l_{p}-x_{p}}{\Delta x_{0}}
$$

Consequently, we will have $\varphi \leq 1$ for the requirement not to strike the rear bumper of vehp, and vice versa. To evaluate the overall hazard state of a specific lane, we believe it would be better not only considered the total offensive time temporally, also the severity of offense spatially and its nonlinear increasing hazard features. For example, a vehicle with the risk factor equaling to four at a particular time is double higher than the risk of two. Thus, we define an exponential function to take into account instantaneous criterion-violated performance of the vehicle operation along the driveway,

$$
f(x)=e^{\alpha(x-1)}-1
$$

In equation (5), the parameter $\alpha$ is determined by the range of risk factor to limit the hazardous weight interval at $(0,1)$. We further introduce $\phi_{i}$ to represent the hazard level of $i^{\text {th }}$ lane, and formula to calculate the Lane Risk Coefficient (LRC) is

$$
\phi_{i}=\sum_{k} \sum_{t_{a}} \varphi\left(t_{a}, v_{e h_{f}}, v e h_{p}\right) f(\varphi), \varphi>1
$$

\subsection{Evaluation}


We further have to choose a proper reaction time and maximum deceleration for the braking process of vehicles. Argument against human reaction time have been investigated and summarized in [7], and it is shown in their studies that reaction time to full braking are all around $2 \mathrm{~s}$ obtained fro $\mathrm{m}$ regardless of simu lators or a test public road. The International Standards Organization [8] reports that maximum permitted rate of deceleration is specified as $5 \mathrm{~m} / \mathrm{s}^{2}$, and longitudinal human-driving braking is substantially less than the maximum deceleration rate of $8.5 \mathrm{~m} / \mathrm{s}^{2}$, even in the situation for crash avoidance or mitigation [9].

The histogram for risk factor $\varphi$ in deceleration of $8.5 \mathrm{~m} / \mathrm{s}^{2}$ to illustrate risk distribution across lanes is shown in Figure 5.

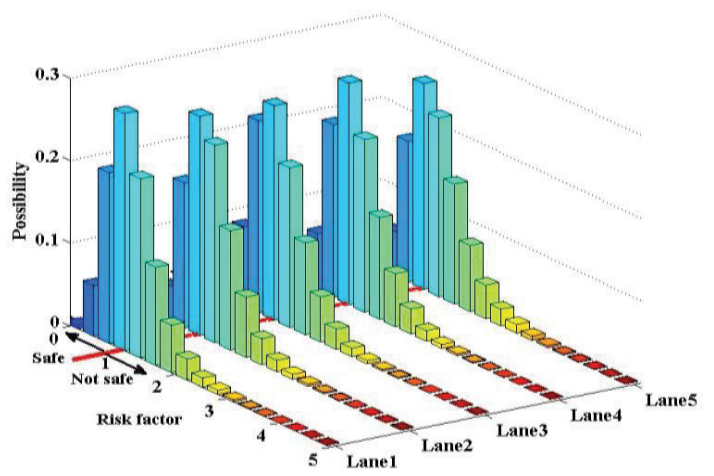

Figure 5. Histograms of risk factor $\varphi$ across lanes.

The demand safe distance of vehicles to recorded distance within the range of zero to five. As seen from Figure 5, the distribution of risk factor over lanes do not have significant differences in shape, which means the safety performance is mainly determined by the amount of ACDA-violation track points of vehicles.

Since it cannot be directly in ferred that how hard all drivers will pressed the brakes to handle with such emergency situation. Thus, we conduct sensitive analys is by changing maximum deceleration vary from $5 \mathrm{~m} / \mathrm{s}^{2}$ to $10 \mathrm{~m} / \mathrm{s}^{2}$. The snapshot of road surface shows a good dry condition, and without loss of generality, it is assumed that all vehicles are the same maximum deceleration rate and reaction delay time. The LRC curve and unsafe proportion of vehicle trace points is shown in Figure 6.

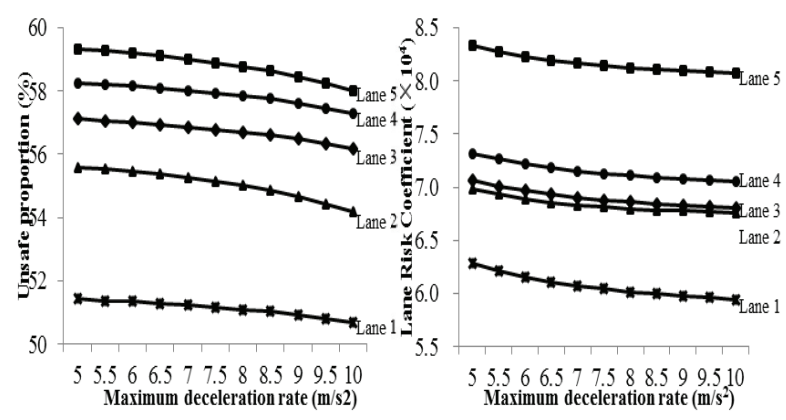

Figure 6. a) Unsafe proportion of trace points violation with ACDA criterion; b) LRC curve with maximum-permitted values of deceleration.
As introduced in equation (4), once drivers have a greater reduction in speed, they are more capable of avoiding collision in critical situation with s maller value of risk factor. It can be clearly observed that the percentage of tracepoints of vehicles driving in unsafe manner increases slightly from around $51 \%$ at Lane 1 to around $59 \%$ at Lane 5.

We can found that LRC decreases with the maximumpermitted deceleration value increasing, which demonstrates that vehicles operating behave more safely. Right lanes carry higher rate of conflicting flows with respect to the left. This indicates that the higher averaged speed of the outside lane, not only causing a more difficult situation for merging process and leading to congestion of a capacity drop, also reduce the safety status because of merging with smaller gaps in the traffic. Interestingly, the values of LRC are quite close in three middle lanes (especially between in Lane 2 and Lane 3), so that we have reason to deduce a similar safely -lane-use pattern between the three lanes.

\section{Conclusions}

It appears completely new that the most-middle lanes are similar utilized under congested conditions compared to two sides of the lanes, as well as the quite close values of LRC to reveals an uniformly safety level between Lane 2 and Lane 3. This may have close relation with relatively unrestrained lane choice in middle lanes. More importantly, since the shoulder Lane 5 is under-utilized but with most hazardous lane use characteristic, toward which to suggest implementing a proper variable speed limit will be beneficial to overall safety performance.

In this paper, many of statistical results are in some extent consistent to a few previous literatures. However, limitation in the availab le reference data did not allow for an extensive quantitative comparison, and as such, the analysis was limited to a qualitative examination of the results and can only explain site-specific traffic in congestion flow. A more complete theory of lane use behavior might also consider such factors as lane and ramp configurations, average vehicle spacing, and lanechanging behavior. Further studies on lane specific behavior and ramp configurations will be summarized in our next study to demonstrate. We also have following insight of this study,

- The study has empirical evidence that demonstrate the use of lane-specific information is a critical factor that should be incorporated with in microscopic traffic simulation models. It is felt that the findings are intended to be relevant to the calibration of microscopic models.

- The lane-by-lane safety assessment reveals that nonuniform flow parameters with significant difference may adversely affect lane-using performance in real traffic. It is hoped that the consideration may lead to a better simulation of traffic conditions and thus aid in the successfuloperation of freeway traffic management.

- Critical for lane level routing analysis once we can fully understand the traffic dynamics on lane level, thus the BPR function or friction factor could be produced based on lane level analysis. 


\section{References}

1. N. Chiabaut, L. Leclercq, C. Buisson, "From heterogeneous drivers to macroscopic patterns in congestion [J]". Transportation Research Part B: Methodological, 44, pp. 299-308 (2010)

2. Highway capacity manual (HCM), Transportation Research Board, Washington D.C (2010)

3. A. Duret, A. Soyoung, B. Christine, "Lane flow distribution on a three-lane freeway: General features and the effects of traffic controls [J]". Transportation Research Part C: Emerging Technologies, 24, pp. 464-464 (2012)

4. A. Pompigna, F. Rupi, "Capacity and Lane Distributional Effects on a Three-Lane Carriageway Section: a Case Study on the Italian Freeway Network", Sidt Scientific Seminar (2015)
5. P. Maravelias, "Tactics for safety and optimization", Advanced highway driving, (2015)

6. B S. Kerner, "Empirical Complex Pattern Formation Caused by Peculiarities of Freeway Infrastructure [M]", The Physics of Traffic, Springer Berlin Heidelberg, 58, pp. 365-372 (2004)

7. D V. Mcgehee, E N. Mazzae, G H S. Baldwin, "Driver Reaction Time in Crash Avoidance Research: Validation of a Driving Simulator Study on a Test Track [J]". Marine Pollution Bulletin, 44, pp. 11571165 (2000)

8. International Standard Organization (ISO), "Full speed range adaptive cruise control system, Standard \#22179, Geneva (2009)

9. DB. Fambro, K. Fitzpatrick and RJ. Koppa, "Determination of stopping sight distance [M]", National cooperative highway research program (NCHRP), National Academy Press (1997) 